\title{
An Optimized Algorithm for Resource Allocation for D2D in Heterogeneous Networks
}

\author{
Abdul Kadir Hamid ${ }^{1}$, Fahd N. Al-Wesabi ${ }^{2,3, *}$, Nadhem Nemri ${ }^{4}$, Ammar Zahary ${ }^{3}$ and Imran Khan \\ ${ }^{1}$ Department of Electrical \& Computer Engineering, University of Sharjah, Sharjah, United Arab Emirates \\ ${ }^{2}$ Department of Computer Science, King Khalid University, Muhayel Aseer, KSA \\ ${ }^{3}$ Faculty of Computer and IT, Sana'a University, Sanaa, Yemen \\ ${ }^{4}$ Department of Information Systems, King Khalid University, Mayahel Aseer, KSA \\ ${ }^{5}$ Department of Electrical Engineering, University of Engineering and Technology, Peshawar, Pakistan \\ ${ }^{*}$ Corresponding Author: Fahd N. Al-Wesabi. Email: falwesabi@kku.edu.sa \\ Received: 18 May 2021; Accepted: 19 June 2021
}

\begin{abstract}
With the emergence of 5G mobile multimedia services, end users' demand for high-speed, low-latency mobile communication network access is increasing. Among them, the device-to-device (D2D) communication is one of the considerable technology. In D2D communication, the data does not need to be relayed and forwarded by the base station, but under the control of the base station, a direct local link is allowed between two adjacent mobile devices. This flexible communication mode reduces the processing bottlenecks and coverage blind spots of the base station, and can be widely used in dense user communication scenarios such as heterogeneous ultra-dense wireless networks. One of the important factors which affects the quality-of-service (QoS) of D2D communications is co-channel interference. In order to solve this problem of co-channel interference, this paper proposes a graph coloring based algorithm. The main idea is to utilize the weighted priority of spectrum resources and enables multiple D2D users to reuse the single cellular user resource. The proposed algorithm also provides simpler power control. The heterogeneous pattern of interference is determined using different types of interferences and UE and the priority of color is acquired. Simulation results show that the proposed algorithm effectively reduced the co-channel interference, power consumption and improved the system throughput as compared with existing algorithms.
\end{abstract}

Keywords: 5G; device-to-device communication; wireless networks; spectrum

\section{Introduction}

With the rapid proliferation in emerging wireless technologies, the sixth-generation $(6 \mathrm{G})$ is considered as one of the most potential candidate which will fulfill the requirements for the future heterogeneous networks [1]. Under the control of the cellular communication system, D2D communication allows terminal users to communicate directly within a certain range by

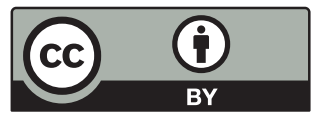

This work is licensed under a Creative Commons Attribution 4.0 International License, which permits unrestricted use, distribution, and reproduction in any medium, provided the original work is properly cited. 
sharing cell resources, and is one of the key technologies of 6G [1-7]. As a new generation of cellular network technology, D2D communication technology has advantages such as improving the utilization of spectrum resources and increasing system throughput, and has become one of the key technologies that have been widely concerned in the industry. However, due to the sharing of cellular network resources, D2D communication causes severe interference to existing cellular systems. Therefore, cellular communication and D2D communication require reasonable cooperative communication to achieve the purpose of interference suppression. At the same time, choosing the way that D2D users and cellular users reuse the same spectrum resources not only saves the precious wireless resources of the cellular network, but also obtains higher spectrum utilization and improves the performance of the cellular network [8-14].

Device-to-Device (D2D) communication enables end users within a short distance to directly transmit data without forwarding through the base station, thereby greatly improving the data transmission rate. In addition, D2D users can also reuse the wireless resources of cellular users in a non-orthogonal manner to improve the utilization of spectrum resources $[15,16]$. Therefore, D2D communication has received extensive attention from the industry in recent years. However, the multiplexing of wireless resources will cause co-channel interference $\mathrm{m}$ in the system. Therefore, how to suppress co-channel interference by allocating wireless resources for D2D users has important practical significance and research value for improving spectrum utilization and meeting users' high-speed data transmission requirements [17-20]. The authors in [21] analyzed three signal demodulation schemes in the uplink based on the outage probability, trying to reduce the influence of $\mathrm{D} 2 \mathrm{D}$ users on the base station through mode selection, and at the same time derive the interference restriction area in the [22] to prevent Adjacent users reuse the same cellular resources. The authors in [23] studied and analyzed the applicability of the LTE power control scheme in D2D systems, and concluded that the hybrid network interference problem must be a combination of power control, mode selection, link adaptation and other technologies. The authors in [24] optimized the transmission power of different users based on system energy efficiency modeling, using sub-optimal algorithms of upper and lower bounds. The authors in [25] combined resource allocation and power control problems and used a novel reverse iterative combined auction method to achieve the optimal goal of system energy efficiency. The authors in [26] proposed a distributed iterative power control algorithm that uses slow-scale path loss measurement to minimize power consumption under the system's target rate constraint. The authors in [27] and [28] deduced the cumulative distribution of signal-to-interference and noise ratio of different users in a hybrid D2D network, laying a foundation for the discussion of power control methods under different QoS requirements. At the same time, the authors in [29] studied the power optimization problem under different resource reuse modes in the followup work, and in-depth deduced the feasible region and closed solution of the system under different constraints. The authors in [30] further studied the system rate maximization problem under the QoS constraint, and the two suboptimal strategies proposed by them did not bring substantial system performance loss. In [31], the authors established interference graphs based on the interference list provided by D2D users, and performed sequential resource allocation based on graph coloring, but this algorithm has higher performance requirements for terminal equipment. The authors in [32] establishes a weighted bipartite graph between D2D users and cellular users, and uses the optimal matching algorithm to allocate wireless resources, which improves the system throughput, but the algorithm complexity is relatively high. In [33], the authors allow a D2D user to reuse the wireless resources of a cellular user, and use optimal matching to minimize co-channel interference in the system. The authors in [34] gave a cognitive spectrum allocation method based on graph coloring theory. This method divides the system bandwidth into multiple sub-channels, 
and then allocates the sub-channels to users, thereby minimizing bandwidth requirements. But this method cannot maximize system throughput. In addition, the existing graph coloring resource allocation algorithms all use D2D users and cellular users as peer users to establish a regular interference graph, and then perform wireless resource allocation. However, the introduction of D2D communication is only an auxiliary communication method for cellular communication, and such wireless resource allocation cannot effectively reflect the importance of users of different communication types. In addition, the traditional graph coloring resource allocation algorithm that only judges the coloring priority based on vertex saturation is far from being able to meet actual communication needs.

Considering the above-mentioned problems, this article proposes a graph-based coloring method for D2D users when multiplexing the downlink of cellular users in the LTE system to communicate, especially in scenarios where communication quality and transmission speed are required in social networks. The weighted priority D2D resource allocation algorithm. Compared with the traditional graph coloring resource allocation algorithm, the advantages of this algorithm are:

(1) In the D2D heterogeneous network, a heterogeneous interference graph is established to reflect the difference in the importance of the two types of users in the network.

(2) The coloring sequence is no longer the previous random coloring or sequential coloring, but a weighted priority coloring.

(3) After the wireless resource allocation is determined, the introduction of simple packet power control can not only further reduce the user's transmission power and reduce the impact of interference, but also dynamically adjust the energy consumption on specific wireless resources.

Finally, combined with the algorithm simulations, we compared and analyze the advantages of the proposed algorithm over other similar algorithms.

\section{System Model}

In the D2D heterogeneous network, there are two types of users in the system: traditional pair of cellular users and short-distance D2D users. The establishment of the system model is shown in Fig. 1. There are a total of $M$ cellular users in the system, which is $C=\left\{C_{1}, C_{2}, \ldots, C_{M}\right\} ; N$ D2D communication pairs, denoted as $D=\left\{D_{1}, D_{2}, \ldots, D_{N}\right\}$. Among them, $C_{i}$ represents $i$ th cellular user, $T_{x_{i}}$ represents the sender user in the $i$-th D2D user pair, and $R_{x_{i}}$ represents the $i$-th receiver user. At the same time, D2D users perform data transmission with the same initial transmission power. In addition, use $L_{c}$ to represent the cellular link set; $L_{d}$ represents the collection of all D2D links; $L_{k}$ represents a set of links using radio resource $k$. In order to improve spectrum utilization, multiple D2D users are allowed to perform data transmission on multiplexed wireless resources of the same cellular user.

Since the LTE system adopts orthogonal frequency division multiplexing (OFDM) technology for downlink, there is no interference between cellular users, and D2D users multiplex the downlink channel resources of cellular users in a non-orthogonal manner, there are two main types of interference in the system: D2D user interference and D2D user-to-cell interference.

The specific interference situation is shown in Fig. 1. Here, $C_{1}, T_{x_{1}}$, and $T_{x_{2}}$ use the same channel resources for data transmission, and the base station will cause co-channel interference to $R_{x_{1}}$ and $R_{x_{2}}$. At the same time, $T_{x_{1}}$ will also cause co-channel interference to $C_{1}$ and $R_{x_{2}}$. In addition, $T_{x_{2}}$ will also cause co-channel interference to $C_{1}$ and $R_{x_{1}}$. It can be seen that the 
interference situation in the D2D heterogeneous network is more complicated. For this reason, it is necessary to allocate spectrum resources reasonably to coordinate co-channel interference, so as to ensure the communication quality of terminal users and improve the spectrum utilization.

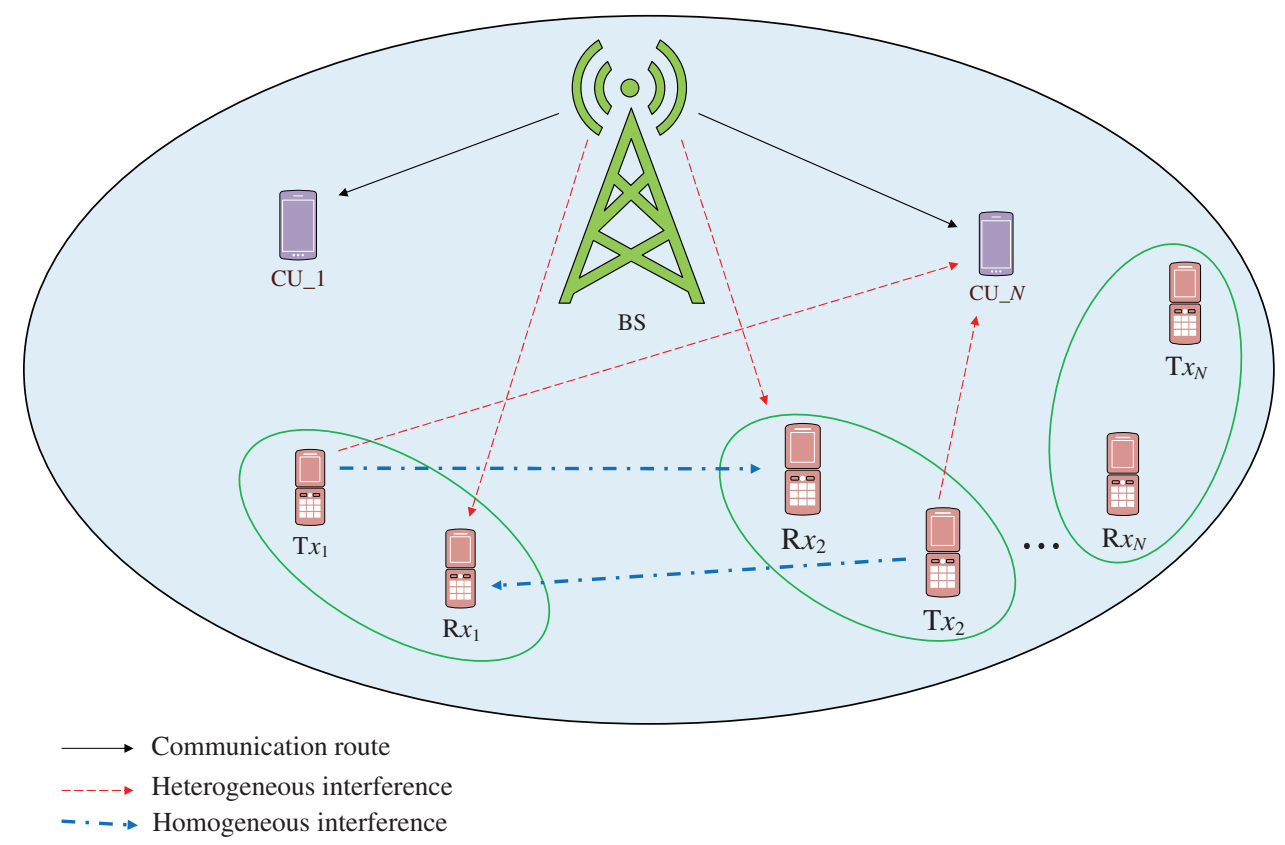

Figure 1: Proposed system model

In addition, assuming that $\left(\varphi_{i}, r_{i}\right)$ is the polar coordinates of user $i$, the distance $d_{i j}$ between user $i$ and user $j$ is expressed in polar coordinates as

$d_{i j}=\sqrt{r_{i}^{2}+r_{j}^{2}-2 r_{i} r_{j} \cos \left(\varphi_{i}+\varphi_{j}\right)}$

where $r_{i}$ represents the distance between user $i$ and the base station; $\varphi_{i}$ represents the azimuth of user $i$. Then the path loss model in the system is as follows. The path loss from the base station to the user is

$p=148+37.6 \log (d[k m])$

The path loss between different users is

$p=148+40 \log (d[k m])$

Among them, $d$ represents the distance between the transceiver ends of the communication link.

\section{Proposed Algorithm}

According to the actual situation, in the D2D heterogeneous network, D2D communication is an auxiliary communication mode. Cellular users are primary users and D2D users are only secondary users. Therefore, the primary and secondary users should be strictly distinguished during interference analysis. In addition, in order to meet the user's normal communication 
quality requirements, the preset SINR threshold for normal communication of cellular users is $\gamma_{1}$, and the SINR threshold for normal communication of D2D users is $\gamma_{2}$. In the weighted priority allocation of resources based on graph coloring, the co-channel interference between communication links and links in the system is abstracted into "vertices" and "edges" in the graph, so as to establish a heterogeneous interference graph $H$. In particular, when building a heterogeneous interference graph, each communication link in the system should be abstracted as a "vertex" of the heterogeneous graph. However, whether there is an "edge" between the two "vertices", it must be combined with the user SINR The threshold is determined by the degree of co-channel interference between users.

\subsection{Generating Heterogeneous Interference Graph}

The "vertex" in the heterogeneous interference graph consists of cellular links and D2D links. Among them, all cellular links constitute vertex set $V_{1}$, and all D2D communication links constitute vertex set $V_{2} . V_{1}$ and $V_{2}$ together form the set $V$ of all vertices in the graph. According to the inter-vertex interference and the user's communication quality requirements, the set of edges $E$ is formed, and the heterogeneous interference graph $H\left(V\left(V_{1}, V_{2}\right), E\right)$ can be obtained. Combining the characteristics of the D2D heterogeneous network, the edges in $H$ are mainly divided into two types: single-type interference edges and mixed-type interference edges. Among them, the single-type interference edge is the edge established for the cellular link that only receives single interference from the D2D link. The mixed interference edge is an edge created by the mixed interference from the cellular link and other D2D links for the interference suffered by the D2D link.

When multiple D2D user pairs use the same radio resource $k$ with the same cellular user in a non-orthogonal manner. The SINR of the cellular user $C_{i}$ is

$\operatorname{SINR}_{C_{i}}=\frac{P_{i, k} G_{B, C_{i}}}{\sum_{j \in L_{D}, j \in L_{k}} P_{T_{x_{j, k}}} G_{T_{x_{j, C}}}+\eta B}$

The SINR of the receiving end of the D2D link such as $R_{x_{j}}$ is

$\operatorname{SINR}_{R_{x_{j}}}=\frac{P_{T_{x_{j, k}}} G_{T_{x_{j}, R_{x_{j}}}}}{\sum_{j \in L_{C}, i \in L_{k}} P_{i, k} G_{B, R_{x_{j}}}+\sum_{j^{\prime} \in L_{D}, j^{\prime} \in L_{k}, j^{\prime} \neq j} P_{T_{x_{j, k}}} G_{T_{x_{j^{\prime}}, R_{x_{j}}}}+\eta B}$

where: $\operatorname{SINR}_{C_{i}}$ represents the SINR of $C_{i} ; P_{i, k}$ represents the transmit power of the base station to $C_{i}$ on channel $k ; G_{B, C_{i}}$ indicates the link gain from the base station to $C_{i} ; P_{T_{x_{j, k}}}$ represents the transmit power of $T_{x_{j}}$ on channel $k ; G_{T_{x_{j}, C_{i}}} ; G_{T_{x_{j}, C_{i}}}$ represents the link gain from $T_{x_{j}}$ to $C_{i} ; \eta$ represents the power of Gaussian white noise; $B$ represents the channel bandwidth.

In addition, the interference generated by the sending end of link $i$ to the receiving end of link $j$ is defined as $I_{i j}$, which is specifically expressed as

$I_{i j}=P_{i} G_{i j}$

where $P_{i}$ represents the transmit power of the transmitting end of link $i$; $G_{i j}$ represents the link gain from the sending end of link $i$ to the receiving end of vertex $j$.

To establish a single-type interference edge, the steps (pseudo code) are as follows in Algorithm 1. 


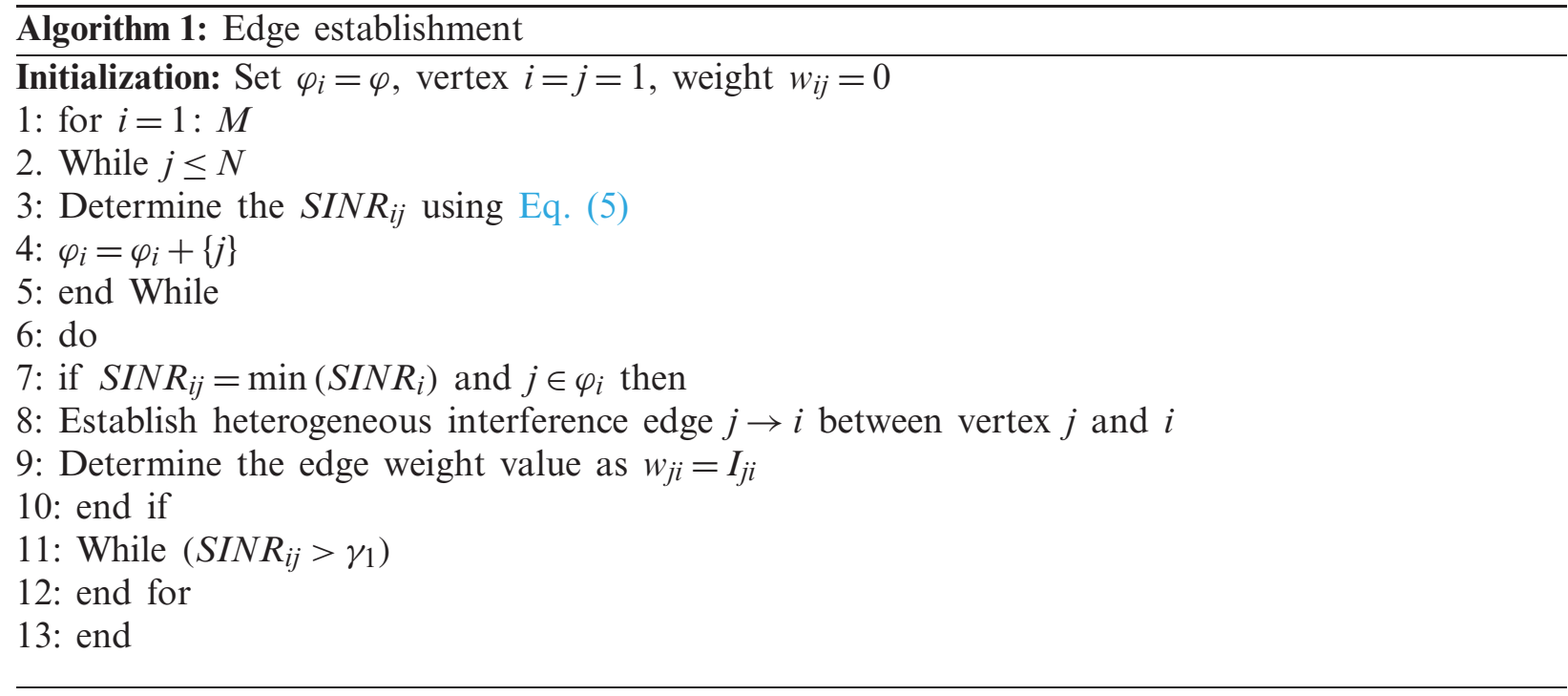

The steps and methods for establishing heterogeneous-type interference edges are similar to those of single-type interference edges. The difference is that in step 3, the SINR is calculated using Eq. (5), and the judgment condition in step 11 is changed to $S I N R_{i j}>\gamma_{2}$. Therefore, it will not be repeated here.

After the single-type and heterogeneous-type interference edges are established, a heterogeneous interference graph is finally obtained. At this time, the $i$ saturation $S(i)$ of the vertex is defined as

$S(i)=\sum_{j \in V, j \neq i} I_{j i}+\sum_{j \in V, j \neq i} I_{i j}$

\subsection{Weighted Priority Resource Allocation}

Assuming that there are two buffer queues at the base station, which store the cellular users and D2D users that initiate resource requests, respectively, and each user corresponds to a data queue to buffer the data to be sent. Without loss of generality, the factors that define the priority of obtaining resources include: (1) Channel quality; (2) Delay of waiting in line for users; (3) The amount of data to be sent by the user; (4) The maximum time delay the user can tolerate. Generally, the higher the priority of the user to obtain resources, the better the channel quality, the longer the user's queuing delay, the more data to be sent, and the lower the user's tolerable waiting delay. Assuming that the time when user $i$ initiates a resource request is recorded as $t_{i, a r r_{\text {_time}}}$, and the time when wireless resources are obtained is recorded as $t_{\text {i,leave_time, }}$ then the waiting delay $T_{i}$ of user $i$ in the queue is

$T_{i}=t_{\text {i,leave_time }}-t_{i, \text { arr_time }}$

When the waiting delay exceeds the maximum tolerable delay, the user will discard the data packet to be sent. In order to prevent users with poor channel quality from being unable to allocate resources for a long time and reduce the fairness of the system, here the delay is taken 
as an important factor that affects the priority of users obtaining resources, and the delay weight of user $i$ is defined as

$W_{i}=e^{\left(\frac{T_{i}}{T_{i, \max }}-1\right)}$

where $T_{i, \max }$ of user $i$ is allowed to accept the maximum delay.

Assuming that the user expects to send the data size as $Q_{i}^{\prime}$, the maximum amount of data that can be buffered in the data queue is $Q_{i, \max }$, so the actual size of data to be sent in the corresponding data queue $Q_{i}$ is expressed as

$Q_{i}=\min \left\{Q_{i}^{\prime}, Q_{i, \max }\right\}$

At the same time, the signal-to-noise ratio is used to measure the channel quality. The larger the signal-to-noise ratio, the better the channel quality. The signal-to-noise ratio at the receiving end of link $i$ is specifically expressed as

$S N R_{i}=\frac{P_{i} G_{i i}}{\eta B}$

Based on the above analysis, the priority for user $i$ to obtain wireless resources is defined as

$D(i)=\frac{Q_{i} \times S N R_{i} \times W_{i}}{\sum Q_{i^{\prime}} \times \sum S N R_{i^{\prime}} \times \sum W_{i^{\prime}}}$

where $D(i)$ represents the priority of user $i$ to obtain resources. The corresponding vertices $i$ and $i^{\prime}$ belong to the vertices of the same set.

When coloring vertices, the user priority and the saturation of the corresponding vertices are considered to determine the coloring order. According to Eqs. (7) and (12), the coloring level $F(i)$ of vertex $i$ in the graph is defined as

$F(i)=D(i) \times \frac{S(i)}{\sum S(i)}$

The current layer color vertex color* needs to meet the conditions as

color $^{*}={ }_{\varepsilon_{i}}^{\max } F(i)$

At the same time, the colored vertices are deleted from the corresponding vertex set, and the uncolored vertices are updated.

Consider that D2D communication is only an auxiliary communication mode. Therefore, when performing coloring resource allocation based on the heterogeneous weighting of graph coloring, it is necessary to color the vertices in the vertex set $V_{1}$ first, and then color the vertices in the set $V_{2}$. The specific coloring steps (pseudo code) are described as follows in Algorithm 2.

In order to evaluate the fairness among D2D users in the system, jains fairness evaluation standards are used such as

$\operatorname{Fair}(n)=\frac{\left(\sum_{i=1}^{n} x_{i}\right)^{2}}{n \sum_{i=1}^{n} x_{i}^{2}}$ 
where Fair (.) represents the fairness of the system; $n$ represents the number of D2D users requesting resources; $x_{i}$ represents the transmission rate of $\mathrm{D} 2 \mathrm{D}$ user $i$.

The D2D system user loss rate is defined as the ratio of the number of users who abandon the queue waiting for resource allocation due to exceeding the maximum time delay to the total number of requested users, which is specifically defined as

$P L R=\frac{n}{N}$

where $n$ represents the number of $\mathrm{D} 2 \mathrm{D}$ users whose waiting delay exceeds the maximum delay; $N$ represents the number of $\mathrm{D} 2 \mathrm{D}$ users requesting resources.

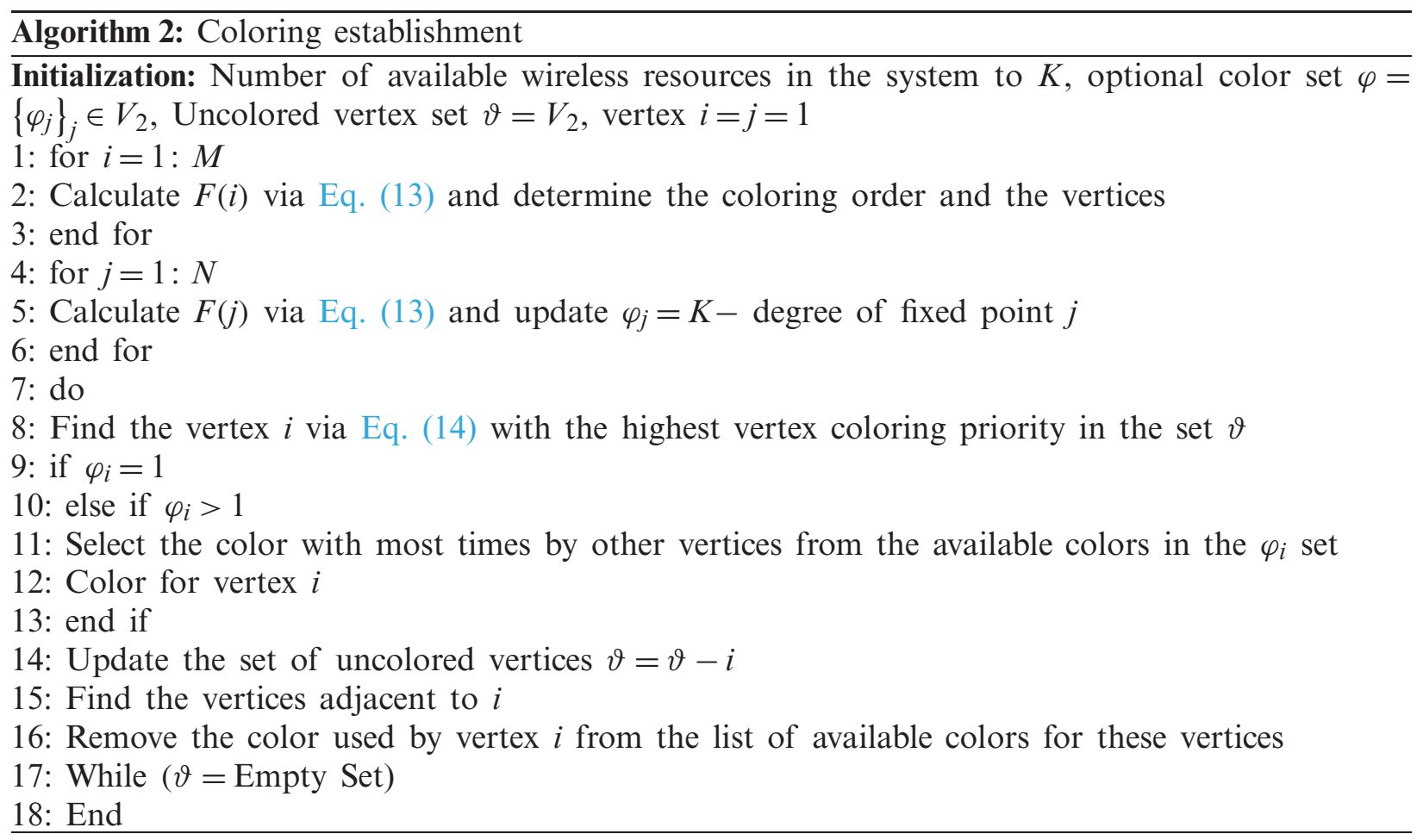

\subsection{Packet Power Control}

In order to reduce the power consumption of the user terminal, on the premise of ensuring the user's minimum SINR requirement, simple power regulation is adopted to minimize the transmission power of the corresponding user. Color the wireless resource allocation results according to the graph, treat all users occupying the same wireless resources as a group, and perform power control on all users in each group, thereby optimizing the user's transmission power.

According to the resource allocation result, calculate the actual SINR of cellular users and D2D users. Combining the SINR threshold $\gamma_{1}$ of $C_{m}$ and the transmit power of D2D users in the same group of $G_{m}$, the lowest transmit power can be obtained as

$$
P_{m}^{\prime}=\frac{\gamma_{1}\left(\sum_{D_{i} \in G_{m}} P_{T_{x_{i}}} G_{T_{x_{i}}, C_{i}}+\eta B\right)}{G_{B, C_{m}}}
$$


where $P_{m}^{\prime}$ represents the transmission power of the base station to $C_{m}$ after power regulation.

Update the transmission power of the base station to $C_{m}$ according to Eq. (17), and calculate the SINR of all D2D users in combination with Eq. (5). Then, update the transmission power of D2D users one by one according to the SINR from descending order. According to the threshold requirement $\gamma_{2}$, the transmit power of the corresponding $T_{x_{n}}$ of the $R_{x_{n}}$ with the largest current SINR of the updated $G_{m}$ is expressed as

$P_{T_{n}}^{\prime}=\frac{\gamma_{2}\left(P_{m}^{\prime} G_{B, C_{m}}+\sum_{D_{i} \in G_{m}} P_{T_{x_{i}}} G_{T_{x_{i}}, R_{x_{i}}}+\eta B\right)}{G_{T_{x_{n}}, R_{x_{n}}}}$

where $P_{T_{n}}^{\prime}$ represents the transmit power of $T_{n}$ after power control; If $T_{i}$ transmission power has been updated, $P_{T_{i}}$ is the updated transmission power, otherwise it is the original power.

For D2D users whose power has not been updated, according to the order of SINR from large to small, continue to use Eq. (18) to update the transmission power, thereby reducing system power consumption.

\section{Simulation Results}

In order to evaluate the impact of the D2D wireless resource allocation algorithm proposed in this paper on system performance, MATLAB simulation is used for verification and analysis. Users are uniformly distributed in the cell, cellular users are distributed within $200 \mathrm{~m}$ of the center of the base station, and D2D users reuse cellular user downlink resources. The waiting time delay of each user is randomly generated during simulation, and the other simulation parameter settings are shown in Tab. 1. Since the location of all users in the cell has a certain randomness, the number of simulations is 500 times and the average is taken so that the data obtained has more reference value. At the same time, the random coloring resource allocation algorithm (RCA), the coloring resource allocation algorithm (DCA) that only considers the delay and the traditional graph coloring resource allocation algorithm (TGCA) are used as references to compare with the proposed resource allocation algorithm, focusing on D2D Performance in terms of system fairness, system throughput, and system power consumption.

Table 1: Simulation parameters

\begin{tabular}{ll}
\hline Parameter & Value \\
\hline Cell radius & $750 \mathrm{~m}$ \\
D2D user pair distance & $10 \sim 25 \mathrm{~m}$ \\
Number of sub-channels & 5 \\
System bandwidth & $5 \mathrm{MHz}$ \\
Number of cellular users & 5 \\
Base station transmission power & $43 \mathrm{dBm}$ \\
D2D user transmit power & $24 \mathrm{dBm}$ \\
Gaussian white noise density & $-174 \mathrm{dBm} / \mathrm{Hz}$ \\
SINR thresholds $\gamma_{1}, \gamma_{2}$ & $12,16 \mathrm{~dB}$ \\
Standard deviation of shadow fading for cellular and D2D links & 10,12 \\
$\alpha, \beta$ & $0.7,12$ \\
\hline
\end{tabular}


Fig. 2 compares the fairness impact of D2D system resource allocation under different algorithms. It can be seen from Fig. 2 that as the number of D2D user's increases, the fairness of the D2D system begins to decrease. Among them, TGCA aims at maximum throughput, and relatively sacrifices more fairness. When there are 80 pairs of D2D users in the system, using the DGCA algorithm can improve scheduling fairness by $5 \%$ compared to the DCA algorithm. This is because the DCA algorithm only considers the delay and does not consider the vertex saturation, and DGCA not only considers the vertex saturation, but also takes the delay as a factor of coloring, which can improve those who are delayed due to poor channel quality to a certain extent. D2D users who cannot get the resources get the priority of the resources, so the fairness of the system is improved.

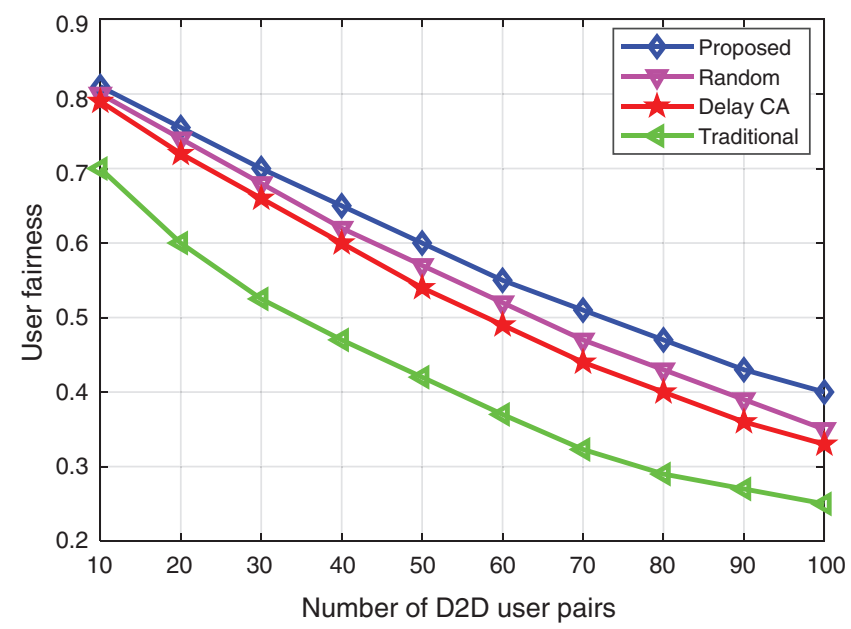

Figure 2: User fairness comparison the proposed and existing algorithms

Fig. 3 compares the influence of the average delay of the D2D system under different shading resource allocation algorithms. It can be seen from Fig. 3 that the average system delay of different algorithms increases as the D2D logarithm of the resource request increases. Among them, compared with the DCA algorithm, the average system delay of the DGCA algorithm is slightly higher by $0.1 \mathrm{~ms}$ when the number of D2D users reaches 50 pairs. However, D2D communication is mainly used for data transmission rather than real-time services, so the $0.1 \mathrm{~ms}$ delay is not perceptible to the user. In addition, combined with the analysis of Figs. 2 and 3 , it can be seen that the DCA algorithm preferentially allocates colored resources to vertices with larger delays, so that the overall system delay is minimized, but the fairness of resource scheduling is seriously sacrificed. Compared with the other two algorithms, the proposed algorithm shows obvious advantages in reducing system delay.

Fig. 4 compares the system throughput under different algorithms. It can be analyzed from Fig. 4 that as the number of D2D users in the system increases, the system throughput also gradually increases. Since the TGCA algorithm only allocates coloring resources based on the vertex saturation, the maximum system throughput is obtained. The proposed algorithm not only considers the vertex saturation, but also improves the coloring priority of the vertices with longer delay, so that fewer packets are discarded. Although the throughput obtained is reduced by an average of $1.5 \%$ compared with TGCA, it is combined with Figs. 2-4 for comprehensive analysis, 
the premise of TGCA to obtain higher throughput is to seriously sacrifice the fairness of user scheduling and to a large extent increase the system delay.

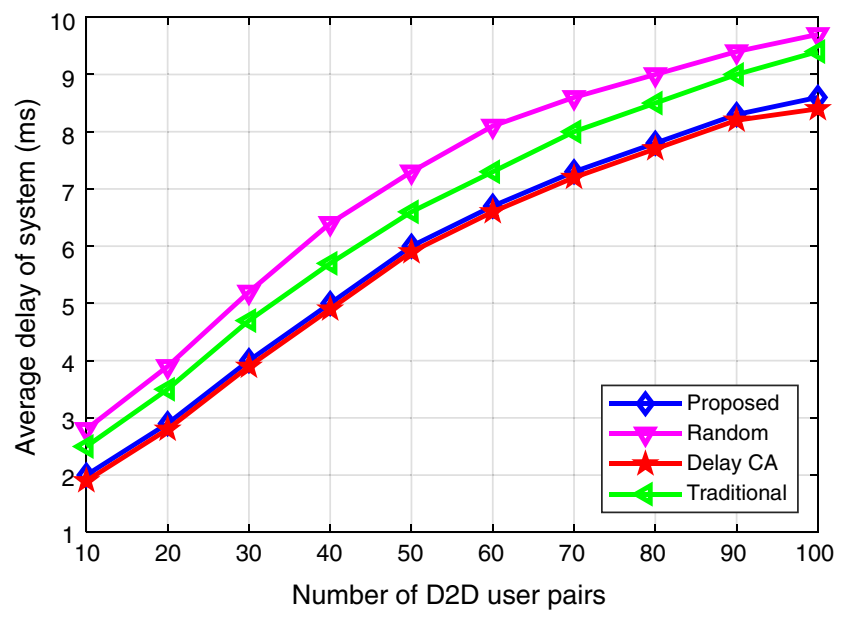

Figure 3: Comparison of system delay of the algorithms

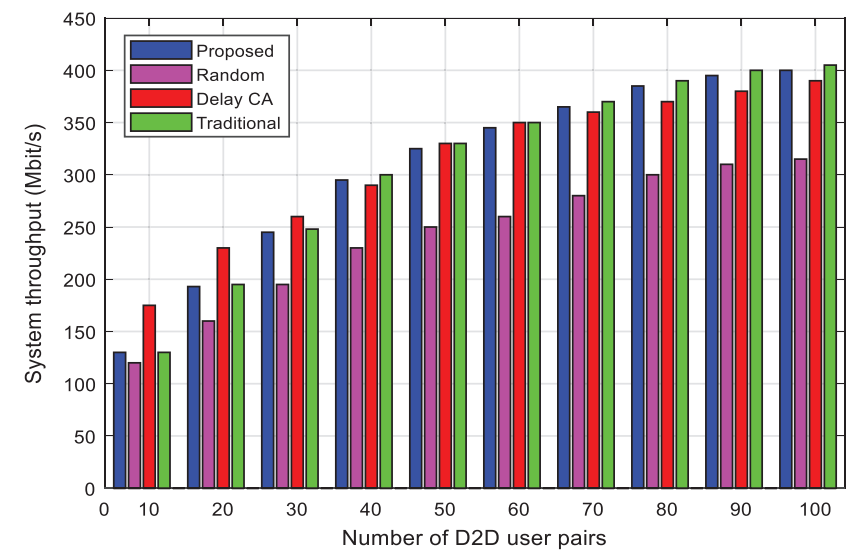

Figure 4: Comparison of the system throughput of the algorithms

Fig. 5 compares the impact of different thresholds on system throughput. The results show that as the D2D user's increases, the system throughput increases, and the larger the threshold, the smaller the system throughput. This is because when the user SINR threshold is not reached, the system access D2D logarithm increases as the requested D2D logarithm increases, thus the system throughput also increases, and the larger the SINR threshold, the less interference the user can bear, allowing access The less the D2D logarithm of, the smaller the system throughput.

Fig. 6 compares the total power consumption of the system with and without power control. When there is no power control, both the base station and the user transmit at a fixed power, and when there is power control, it is based on the minimum user SINR requirement. As can be seen from Fig. 6 that the total power consumption of the system is lower when using the power control as compared with no power control. 


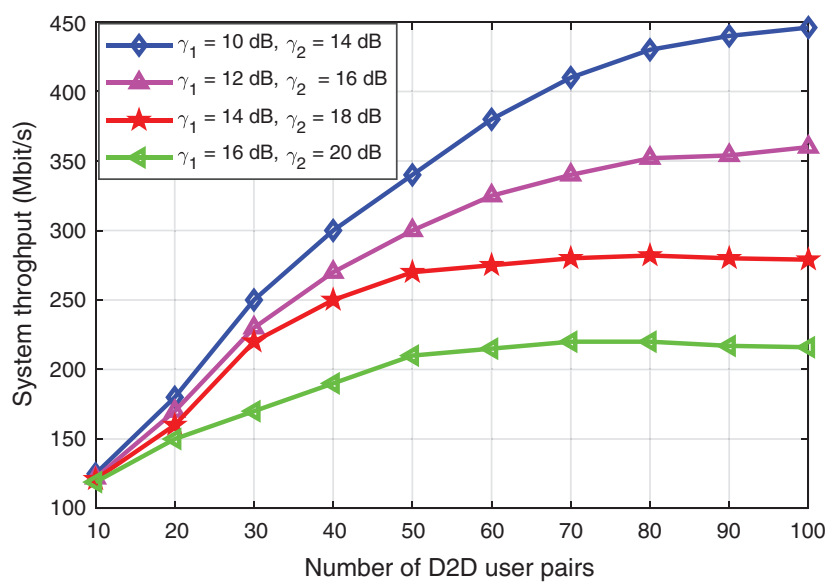

Figure 5: Impact of SINR thresholds on system throughput performance

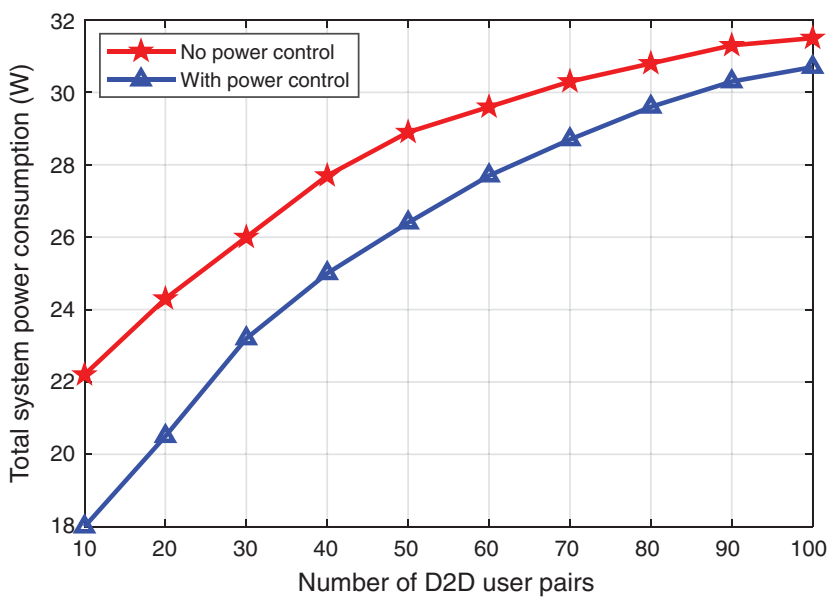

Figure 6: Impact of power control on the system power consumption

\section{Conclusions}

In the TD-LTE system, suitable downlink radio resources of cellular users are selected for multiplexing for D2D users. First, establish a heterogeneous interference graph according to the degree of interference between users with different communication types. The weighted priority coloring order is calculated by further combining the vertex saturation, waiting delay, channel quality, and data volume. Finally, the wireless resource is allocated according to the graph coloring algorithm. Theoretically, the deficiencies of the existing graph coloring resource allocation algorithm are improved, and the maximum throughput performance of the system is achieved. In fact, the factors that affect the allocation of wireless resources are fully considered, which has strong practical application value. Simulation shows that the proposed algorithm has the best overall performance in terms of system average delay, packet loss rate and system throughput, and it also reduces the total power consumption of the system. Further research as an extension to this research study is to consider the cache-throughput analysis and deploy the proposed algorithm in UDNs scenarios. 
Acknowledgement: The author extends his appreciation to the Deanship of Scientific Research at King Khalid University for funding this work under grant number (RGP.2/23/42), Received by Fahd N. Al-Wesabi. www.kku.edu.sa.

Funding Statement: This work is supported by Suranaree University for Technology research and development fund.

Conflicts of Interest: The authors declare that they have no conflicts of interest to report regarding the present study.

\section{References}

[1] Y. Sun, J. Liu, J. Wang, Y. Cao and N. Kato, "When machine learning meets privacy in 6G: A survey," IEEE Communications Surveys \& Tutorials, vol. 22, no. 4, pp. 2694-2724, 2020.

[2] T. Huang, W. Yang, J. Wu, J. Ma, X. Zhang et al., "A survey on green 6G network: Architecture and technologies," IEEE Access, vol. 7, pp. 175758-175768, 2019.

[3] S. Zhang, J. Liu, H. Guo, M. Qi and N. Kato, "Envisioning device-to-device communications in 6G," IEEE Networks, vol. 34, no. 3, pp. 86-91, 2020.

[4] M. A. Garadi, A. Mohammed, A. K. Ali, X. Du, I. Ali et al., "A survey of machine and deep learning methods for internet of things (IoT) security," IEEE Communications Surveys \& Tutorials, vol. 22, no. 3, pp. 1646-1685, 2020.

[5] S. Li, L. Li, B. Xu, Y. Feng and H. Zhou, "Research of a reliable constraint algorithm on MIMO signal detection," International Journal of Embedded Systems, vol. 12, no. 2, pp. 13-26, 2020.

[6] J. Gojal, E. Monteiro and J. S. Silva, "Security for the internet of things: A survey of existing protocols and open research issues," IEEE Communications Surveys \& Tutorials, vol. 17, no. 3, pp. 1294-1312, 2015.

[7] K. Tange, M. D. Donno, X. Fafoutis and N. Dragoni, "A systematic survey of industrial internet of things security: Requirements and foq computing opportunities," IEEE Communications Surveys \& Tutorials, vol. 22, no. 4, pp. 2489-2520, 2020.

[8] S. Bashir, M. H. Alsharif, I. Khan, M. A. Albreem, A. Sali et al., "MIMO-terahertz in 6G nanocommunications: Channel modeling and analysis," Computers Materials \& Continua, vol. 66, no. 1, pp. 263-274, 2020.

[9] A. Silva, S. Teodore, R. Dinis and A. Gameiro, "Iterative frequency-domain detection for IA-precoded MC-CDMA systems," IEEE Transactions on Communications, vol. 62, no. 4, pp. 1240-1248, 2014.

[10] A. Silva, J. Assucao, R. Dinis and A. Gameiro, "Performance evaluation of IB-DFE based strategies for SC-FDMA systems," EURASIP Journal on Wireless Communications and Networking, vol. 13, no. 1, pp. 1-10, 2013.

[11] D. Castanheira, A. Silva, R. Dinis and A. Gameiro, "Efficient transmitter and receiver designs for SC-FDMA based heterogeneous networks," IEEE Transactions on Communications, vol. 63, no. 7, pp. 2500-2510, 2015.

[12] D. Castanheira, A. Silva and A. Gameiro, "Set optimization for efficient interference alignment in heterogeneous networks," IEEE Transactions on Wireless Communications, vol. 13, no. 10, pp. 5648-5660, 2014.

[13] S. Teodoro, A. Silva, R. Dinis, F. Barradas, P. M. Cabral et al., "Theoretical analysis of nonlinear amplification effects in massive MIMO systems," IEEE Access, vol. 7, pp. 172277-172289, 2019.

[14] F. Jameel, T. Ristaniemi, I. Khan and B. M. Lee, "Simultaneous harvest-and-transmit ambient backscatter communications under Rayleigh fading," EURASIP Journal on Wireless Communications and Networking, vol. 19, no. 1, pp. 1-9, 2019.

[15] Q. Alsafasfeh, O. A. Saraereh, A. Ali, L. A. Tarawneh, I. Khan et al., "Efficient power control framework for small-cell heterogeneous networks," Sensors, vol. 20, no. 5, pp. 1-14, 2020. 
[16] K. M. Awan, M. Nadeem, A. S. Sadiq, A. Alghushami, I. Khan et al., "Smart handoff technique for internet of vehicles communication using dynamic edge-backup node," Electronics, vol. 9, no. 3, pp. $1-17,2020$.

[17] W. Shahjehan, S. Bashir, S. L. Mohammed, A. B. Fakhri, A. A. Isaiah et al., "Efficient modulation scheme for intermediate relay-aided IoT networks," Applied Sciences, vol. 10, no. 6, pp. 1-12, 2020.

[18] B. M. Lee, M. Patil, P. Hunt and I. Khan, "An easy network onboarding scheme for internet of things network," IEEE Access, vol. 7, pp. 8763-8772, 2018.

[19] O. A. Saraereh, A. Alsaraira, I. Khan and B. J. Choi, "A hybrid energy harvesting design for on-body internet-of-things (IoT) networks," Sensors, vol. 20, no. 2, pp. 1-14, 2020.

[20] T. Jabeen, Z. Ali, W. U. Khan, F. Jameel, I. Khan et al., "Joint power allocation and link selection for multi-carrier buffer aided relay network," Electronics, vol. 8, no. 6, pp. 1-15, 2019.

[21] H. Min, W. Seo, J. Lee, S. Park and D. Hong, "Reliability improvement using receive mode selection in the device-to-device uplink period underlaying cellular networks," IEEE Transactions on Wireless Communications, vol. 10, no. 2, pp. 413-418, 2011.

[22] H. Min, J. Lee, S. Park and D. Hong, "Capacity enhancement using an interference limited area for device-to-device uplink underlaying cellular networks," IEEE Transactions on Wireless Communications, vol. 10, no. 12, pp. 3995-4000, 2011.

[23] S. Noreen, N. Saxena and A. Roy, "Incentive and penalty mechanism for power allocation in cooperative D2D-cellular transmissions," Electronics, vol. 9, no. 3, pp. 1-13, 2019.

[24] X. Song, S. Xu, Z. Xie and X. Han, "Joint optimal power allocation and relay selection scheme in energy harvesting two-way relaying network," Future Internet, vol. 11, no. 2, pp. 1-12, 2019.

[25] W. Fairan, X. Chen, S. Lingyan, Z. Han and B. Zhang, "Energy-efficient radio resource and power allocation for device-to-device communication underlaying cellular networks," in IEEE Int. Conf. on Wireless Communications \& Signal Processing (WCSP), Huangshan, China, pp. 1-6, 2012.

[26] M. A. Rahman, Y. Lee and I. Koo, "Energy-efficient power allocation and relay selection schemes for relay-assisted D2D communications in 5G wireless networks," Sensors, vol. 18, no. 9, pp. 1-24, 2018.

[27] Z. Lin, H. Song and D. Pan, "A joint power and channel scheduling scheme for underlay D2D communications in the cellular network," Sensors, vol. 19, no. 21, pp. 1-19, 2019.

[28] M. Ertuk, S. Mukherjee, H. Ishii and H. Arslan, "Distribution of transmit power and SINR in deviceto-device networks," IEEE Communications Letters, vol. 17, no. 2, pp. 273-276, 2013.

[29] C. H. Yu, K. Doppler, C. B. Ribeiro and O. Tirkkonen, "Resource sharing optimization for device-todevice communication underlaying cellular networks," IEEE Transactions on Wireless Communications, vol. 10, no. 8, pp. 2752-2763, 2011.

[30] W. Jianheng, Z. Daohua and Z. Chunming, "Resource sharing of underlaying device-to-device and uplink cellular communications," IEEE Communications Magazine, vol. 17, no. 6, pp. 1148-1151, 2013.

[31] C. Lee, S. M. Oh and A. S. Park, "Interference avoidance resource allocation for D2D communication based on graph-coloring," in IEEE Int. Conf. on Information and Communication Technology Convergence (ICTC), Busan, South Korea, pp. 895-896, 2014.

[32] H. Zhang, T. Wang, L. Song and Z. Han, "Radio resource allocation for physical-layer security in D2D underlay communications," in IEEE Int. Conf. on Communications (ICC), Sydney, Australia, pp. 2319-2324, 2014.

[33] J. Han, Q. Cui, C. Yang and X. Tao, "Bipartite matching approach to optimal resource allocation in device to device underlaying cellular network," Electronics Letters, vol. 50, no. 3, pp. 212-214, 2014.

[34] I. Tab, Z. Feng and W. Liu, "Graph coloring based spectrum allocation for femtocell downlink interference mitigation," IEEE Wireless Communications \& Networking Conf., Cancun, Mexico, pp. 1248-1252, 2011. 